\title{
Nontuberculous Mycobacteria in China-incidence and Antimicrobial Resistance Spectrum From a Nationwide Survey
}

\section{Chunfa Liu}

Chinese Center for Disease Control and Prevention

\section{Yimeng Song}

Beijing Hospital

\section{Wencong He}

National Institute for Communicable Disease Control and Prevention

\section{Dongxin Liu}

The Third People's Hospital of Shenzhen

Ping He

National Institute for Communicable Disease Control and Prevention

Jingjing Bao

Inner Mongolia Medical University

\section{Xinyang Wang}

Harbin Medical University

Yanming $\mathrm{Li}$

Beijing Hospital

Yanlin Zhao ( $\nabla$ zhaoyl@chinacdc.cn )

National Center for Tuberculosis Control and Prevention, Chinese Center for Disease Control and Prevention

\section{Research Article}

Keywords: NTM, Pulmonary Disease, Prevalence, Drug Resistance

Posted Date: February 9th, 2021

DOl: https://doi.org/10.21203/rs.3.rs-197161/v1

License: (a) (i) This work is licensed under a Creative Commons Attribution 4.0 International License. Read Full License 


\section{Abstract}

Background: Information on prevalence and resistance spectrum of nontuberculous mycobacteria (NTM) in China is mainly based on regional or local data. A national survey of NTM pulmonary disease was carried out based on acid-fast positive sputum samples collected in 2013.

Methods: Sputum samples collected from enrolled presumptive cases were cultured using L-J medium in 72 nationwide tuberculosis surveillance sites from the 31 Chinese mainland provinces and isolates were sent to national tuberculosis reference laboratory (NTRL). The species of re-cultured strains were identified by MALDI-TOF MS and minimal inhibitory concentrations (MICs) were determined to evaluate the drug susceptibility of NTM isolates.

Results: Of 4917 mycobacterial isolates cultured, 317 (6.4\%, 95\% Cl, 5.8\% to 7.2\%) were confirmed as NTM, among which 287 (7.7\%, $95 \% \mathrm{Cl}, 6.9 \%$ to $8.6 \%)$ were from the southern region. In inland and coastal China, $87.7 \%(95 \% \mathrm{Cl}, 78.7 \%$ to $93.2 \%)$ and $50.0 \%(95 \% \mathrm{Cl}$, $43.7 \%$ to $56.3 \%$ ) of isolates, respectively, were slow growing mycobacteria (SGM), with the remaining rapid growing mycobacteria (RGM). A total of 29 species were detected, $M$. abscessus had higher clarithromycin-inducible resistance rates than $M$. massiliense (65.67\% vs $2.22 \%$ ). M. kansasii was present lower resistance rates in linezolid and moxifloxacin than Mycobacterium aviumintracellulare complex and other SGM.

Conclusions: More NTM pulmonary disease was observed in southern and coastal China $(p<0.01)$. SGM was widely distributed, and more RGM are present in southern and coastal China $(\mathrm{p}<0.01)$. The antimicrobial resistance spectrum of different NTM species was significant different, accurate species identification would be facilitated to NTM pulmonary disease treatment.

\section{Background}

NTM pulmonary disease (NTM-PD) is a severe progressive illness caused by NTM, that requires complicated treatment with multiple anti-mycobacterial drugs for more than 12 months [1]. Several studies have reported increasing incidences of pulmonary NTM isolation, likely due to socioeconomic and medical progress, including advances in radiological diagnostics, which have improved the rate of detection of pulmonary abnormalities [2]; however, the increase in the aging population with chronic lung diseases or who are immunocompromised may have also contributed to the emergence of NTM-PD. Mortality from pulmonary disease caused by NTM is higher than that attributable to Mycobacterium tuberculosis (MTB), due to inappropriate treatment and high rates of therapy failure [3]. A meta-analysis indicted that proportion of NTM among mycobacterial isolates in China was higher than most regions in whole world except that in Northern India [4]. Although several studies of NTM epidemiology in China have been reported, the NTM isolates were primarily obtained from medical institutions and comprised incomplete samples [3,5]. Therefore, precise incidence and prevalence data is not available.

Antimicrobial agent drug susceptibility testing (DST) are essential for the treatment of infections, and are important for guiding clinical treatment. In addition, some acid-fast staining bacteria those are not mycobacteria, such as Gordonia and Nocardia, have similar colony morphology on Lowenstein-Jensen (L-J) media. Furthermore, most NTM are inherently resistant to standard anti-tuberculosis drugs and different species exhibit varying resistance phenotypes [6]. Therefore, an accurate and rapid method for NTM identification is particularly important. In view of the differences in local development, the national information associated with NTM prevalence and resistant spectrum in China was obtained by meta-analysis that data was collected in regional areas, which lead a lack of unified methods using for species identification and DST $[4,7]$.

In this study, matrix-assisted laser desorption ionization-time of flight mass spectrometry (MALDI-TOF MS), a technology with low time consumption, modest costs, and high accuracy [8, 9], was used to screening NTM from cultured acid-fast clinical strains isolated from sputum. MICs detected by broth microdilution method were measured for RGM and SGM among the NTM isolates. The objectives of the survey were to determine the proportion of NTM in isolated mycobacteria and to determine their susceptibility to various antimicrobial drugs recommended by the United States Clinical and Laboratory Standards Institute (CLSI) in China mainland.

\section{Methods}

\section{Study Subjects.}

A total of 5487 isolates from patients with suspected acid-fast bacilli-positive tuberculous were collected at 72 nationwide tuberculosis surveillance stations in 2013 and sent to NTRL (Fig. 1). At least one site among the 72 centers from which strains were collected was in 
each of the 31 provinces and municipalities of China. The number of centers assigned to each province and municipality was proportional to the number of new smear-positive cases reported by that province, relative to the previously reported total number of cases nationwide [10].

\section{Study Process.}

All strains were re-cultivated using L-J medium in NTRL; species identification was used to culture positive strains by MALDI-TOF MS after 4 weeks. Identified NTM species were confirmed by sequencing of partial genes (including 16S rRNA, hsp65, rpoB, and ITS). DSTs were performed using the broth microdilution method, with Sensititre ${ }^{\circledR}$ SLOMYCO plates for SGM and a panel of 13 drugs, and Sensititre ${ }^{\circledR}$ RIPMYCOI plates and 15 drugs for RGM (TREK Diagnostic Systems, Cleveland, USA). Using an Ultrasonic Milling Instrument (TB Healthcare, China), $0.5 \mathrm{McF}$ arland bacterial suspensions were prepared from colonies grown on L-J culture medium. Suspensions were diluted 100 -fold by adding $100 \mu \mathrm{L}$ of $0.5 \mathrm{McF}$ arland suspensions to $10 \mathrm{~mL}$ of Mueller-Hinton broth, with or without oleic acidalbumin-dextrose-catalase. Aliquots $(100 \mu \mathrm{L})$ of standard $1.5 \times 10^{5} \mathrm{CFU} / \mathrm{mL}$ inoculum were distributed into each well using a semiautomated Sensititre ${ }^{\circledR}$ Auto-inoculator (Thermo Fisher, Scientific Inc., USA). To prevent evaporation and skipped wells during incubation, plates were accurately and adequately sealed with adhesive membranes, and incubated at the recommended temperature [11]. MIC was defined as the lowest concentration without obvious visible bacteria growth compared with positive controls, and was measured by two readers, aided by a Vizion ${ }^{\mathrm{TM}}$ Digital viewing system. Mycobacteriumabscessus (ATCC 19977) and Mycobacteriumintracellulare (ATCC 13950) were used as quality control samples in every batch of DSTs.

\section{Statistical Analysis:}

The $95 \%$ confidence interval for proportions was calculated according to the Wilson procedure, with correction for continuity, as described by Robert Newcombe, derived from a procedure outlined by E. B. Wilson [12]. All theoretical numbers $T \geq 5$ and total sample size $N \geq 40$ were tested by Pearson Chi-square Test. If the theoretical number $T<5$ but $T \geq 1$, and $N \geq 40$, the Continuous Corrected Chisquare Test of Association is used for testing. If there are theoretical numbers $T<1$ or $N<40$, Fisher Exact Probability Test will be used. $\mathrm{P}<0.01$ defined as statistically significant.

\section{Results}

\section{The ratio of Pulmonary NTM diseases}

A total of 5487 isolates were cultivated using L-J medium; recovery failed for 284 isolates. All cultured isolates were identified by MALDITOF MS and 286 strains were not mycobacteria. Finally, we obtained 317 NTM isolates from 4917 mycobacterium strains, according to the results of MALDI biotyping. NTM species were confirmed by sequencing of partial genes (including 16S rRNA, hsp65, rpoB, and ITS); 5 strains were identified as Mycobacterium tuberculosis complex (MTBC) and another 5 strains identified as MTBC by MALDI-TOF MS were classified as NTM based on whole gene sequencing in another ongoing research program. Finally, we identified 317 NTM strains from 31 provinces in China (Fig. 1).

As shown in Table 1, of 317 NTM strains, $287(7.7 \%, 95 \% \mathrm{Cl}, 6.9 \%$ to $8.7 \%)$ of total isolates were detected in the southern region and the NTM infection rate in the southern region was higher than that in the northern region $(7.7 \%$ vs. $2.5 \%, p<0.01)$. In stratified analysis, the ratio of NTM infection varied according to geographic area (Table 1): the higher ratio was $10.7 \%(95 \% \mathrm{Cl}, 9.5 \%$ to $12.1 \%)$ for the coastal region than $3.0 \%(95 \% \mathrm{Cl}, 2.4 \%$ to $3.7 \%)$ in the inland region. Prevalence rates in individual provinces are presented in Figure 2 . The composition ratio of RGM to SGM in northern and southern China differed markedly $(p<0.01)$. Significantly more RGM were present in coastal than inland China $(P<0.01)$.

\section{Spectrum of NTM species}

As showed in Fig.3, a total of 29 species were detected, including 19 SGM and 10 RGM; 27 species were detected in southern China and only 7 species were observed in northern China. The five most frequently isolated NTM, accounting for $88.5 \%$ of all NTM species, belonged to the Mycobacteriumabscessus complex (MABC) $(36.0 \%, 95 \% \mathrm{Cl}, 30.7 \%$ to $41.5 \%)$, the Mycobacteriumavium-intracellulare complex (MAC) $(34.1 \%, 95 \% \mathrm{Cl}, 28.9 \%$ to $39.6 \%)$, M.kansasii $(9.8 \%, 95 \% \mathrm{Cl}, 6.8 \%$ to $13.7 \%)$, M.paragordonae $(5.4 \%, 95 \% \mathrm{Cl}, 3.3 \%$ to $8.6 \%)$, and M.lentiflavum (3.2\%, $95 \% \mathrm{Cl}, 1.6 \%$ to $5.9 \%)$. The MAC included seven subspecies: M.avium subsp. hominissuis (1 isolate), M.avium subsp. vulneris (2 isolates), M.avium (2 isolates), M.avium subsp. marseillense (16 isolates), M.intracellulare (82 isolates), 
M.intracellulare subsp. yongonense (4 isolates), and M. chimaera (1 isolate). The MABC included three subspecies: M. abscessus (67 isolates), M.bolletii (2 isolates), and M.massiliense (45 isolates).

Next, we analyzed the geographical distribution of the three most frequent NTM species, and found that 114 MABC isolates were obtained from 6 provinces, with 113 strains distributed in southern China; in particular, 93 isolates were from Guangdong province. Further $108 \mathrm{MAC}$ isolates were from 16 provinces (all 317 isolates were obtained from 21 provinces) with no obvious regional differences. Although only 31 M.kansasii were obtained from 11 provinces, the reginal distribution was wide.

\section{Drug Susceptibility Testing Results}

RGM were classified as susceptible, intermediate, or resistant, depending on the MIC values obtained, according to the CLSI criteria [13]. The distributions of the corresponding degrees of susceptibility, including MIC50/MIC90 values, are presented in Table 2. Amikacin was the most active drug against RGM. The resistance rate to amikacin was $4.69 \%(6 / 128)$, while the resistance rate to cefoxitin in RGM was $18.90 \%$ (24/127). For clarithromycin, 14.96\% (19/127) of RGM were resistant at both day 3 and day 14, corresponding to acquired resistance to clarithromycin, while a total of $38.58 \%$ (49/127) of RGM were susceptible on day 3 , but resistant on day 14 , indicating inducible resistance to clarithromycin. The resistance rate to linezolid was $34.65 \%$ for RGM. Resistance rates to imipenem, tobramycin, doxycycline, cefepime, trimethoprim/sulfamethoxazole (TMP-SMX), minocycline, moxifloxacin, ciprofloxacin, ceftriaxone, and amoxicillin/clavulanic acid were high for RGM. As shown in Table 3, comparison of drug susceptibility patterns among the major MABC species, M.abscessus and M.massiliense, indicated a significant difference in clarithromycin-inducible resistance rates $(65.67 \%$ vs $2.22 \%)$ between the two species.

We also obtained MIC data for 189 SGM isolates. The current standard (CLSI M24, 3rd edition) [11] includes recommendations for DST of SGM, including the MAC, M. kansasii, and SGM other than MAC and M. kansasii. Data on the susceptibility of the 108 MAC isolates to 4 antimicrobial agents are presented in Table 4. Clarithromycin was the most active drug against MAC, with a 4.63\% (5/108) resistance rate, while the resistance rate to amikacin was $10.19 \%$ (11/108). Clarithromycin and amikacin, which are recommended by CLSI as firstline antimicrobials to treat MAC infections, showed excellent bacteriostatic effects in vitro. The resistance rates of MAC isolates to the second-line antimicrobials, linezolid and moxifloxacin, were $66.36 \%$ and $47.66 \%$, respectively. Data on the susceptibility of $31 \mathrm{M}$.kansasii isolates to 9 antimicrobial agents is presented in Table 5. No M.kansasii isolates were resistant to clarithromycin, amikacin, or moxifloxacin. The rates of resistance to other first- and second-line antimycobacterial drugs was lower than $50 \%$, other than those of doxycycline $(77.42 \%, 24 / 31)$ and TMP-SMX $(51.61 \%, 16 / 31)$. Data on the susceptibility of 50 SGM other than MAC and M. kansasii to 9 antimicrobial agents is presented in Table 6, the rates of resistant to amikacin, clarithromycin and rifabutin is lower than $10 \%$, followed by moxifloxacin $(26 \%, 13 / 50)$ and linezolid $(38 \%, 19 / 50)$. As shown in Table 7 , comparison of drug susceptibility patterns among SGM, M. kansasii indicated lowest resistant rate in linezolid and moxifloxacin, followed by SGM other than MAC and M. kansasii; MAC showed highest resistant rate in linezolid and moxifloxacin among SGM.

\section{Discussion}

This study demonstrates that, among all myco-bacteriological culture-positive pulmonary disease cases, $6.4 \%$ were NTM, based on nationwide surveillance of drug-resistant tuberculosis. Pulmonary NTM infection was more frequent in southern China, particularly southern coastal areas with high humidity. The most prevalent SGM was the MAC, which comprised seven subspecies, among which, M.intracellulare was predominant and distributed widely across northern and southern China. The most prevalent RGM was the MABC, comprising three subspecies, with M.abscessus the predominant subspecies, and mainly distributed in southern China. The results of DST indicated that the drug-resistance spectrum varied greatly across different strains and subspecies. NTM showed relatively low resistance rates to macrolides and amikacin in vitro.

Distinguishing NTM from MTBC infection is of great clinical significance, as it can direct accurate and rapid clinical treatment [14-16]. The screening method used for NTM species is based on p-Nitrobenzoic acid, a time-consuming and difficult method, which most reports from China indicate can inhibit $M$. tuberculosis complex growth [17]. The laboratory diagnosis methods used for identification of mycobacterial species have evolved over the decades [18]. With the development of several extraction methods that enhance the amount of bacterial proteins available for MALDI-TOF MS identification, and the increasing amount of mycobacteria data available in commercial databases, MALDI-TOF MS technology has been implemented for NTM identification in many laboratories [8, 19]. Several studies have demonstrated that this method can achieve more than $95 \%$ agreement with results from DNA sequencing of variable genomic regions (including the $16 S$ rRNA, $h s p 65, r p o B$, and ITS genes) $[5,20]$. In our research, we obtained a $98.4 \%$ NTM detection rate and achieved $93.4 \%$ agreement with $16 S$ rRNA, hsp65, ITS, and rpoB gene sequencing. Although we could not identify NTM strains that 
were not contained in the Bruker MBT strains database, MALDI-TOF MS was able to identify most clinically relevant NTM in a rapid, reliable, and inexpensive manner.

The overall NTM pulmonary infection rate was approximately $6 \%$ in our study, similar to that reported in a systematic review and metaanalysis of NTM infections, which demonstrated that the prevalence of NTM infections among patients with suspected tuberculosis was $6.3 \%$ in mainland China [7]. The geographic variability in both the prevalence of NTM infections and mycobacterial species composition was clearly demonstrated by our study. A previous investigation in southern-central China demonstrated that the NTM infection rate is $4.0 \%$, with the two most prevalent species the M.avium-intracellulare and Mycobacterium chelonae-abscessus complexes [6], while a report from Shanghai found an overall rate of NTM isolation from mycobacterial culture-positive patients of 5.9\%, with M.kansasii the most frequently identified species, with an increasing trend from $3.0 \%$ in 2008 to $8.5 \%$ in 2012 [21]. In our study, the most frequent species in Shanghai province was also M.kansasii (7/11 isolates), with a further increase in the NTM prevalence rate to $11 \%$ in 2013. In another study in Guangdong and Shanghai provinces, M.intracellulare was the most commonly isolated NTM in Shanghai, while M.abscessus was the most frequently isolated species in Guangzhou [22]. Some reports from eastern and northern regions of China have demonstrated NTM prevalence rates of around $2.0 \%-3.0 \%$, with M.intracellulare the predominant species, followed by $M$. abscessus [5, 21-23]. In our study, NTM infection was more prevalent in southern than northern China and more frequent in eastern than western China. The most epidemic NTM species were MAC, which was widely distributed, and MABC which is mainly distributed in southeastern China. In addition, we isolated 16 M.marseillense strains of the MAC from sputum samples. Pulmonary disease caused by M. marseillense warrants increased attention, as it is infrequently reported [24, 25].

In addition to Mycobacteria spp., we also identified some acid-fast-staining-positive non-mycobacteria. As shown in Figure 1, we randomly selected 60 non-mycobacteria from 286 contaminated or other species for species identification, including: 15 Gordonia, 2 Nocardia, 2 Streptococcus, and 1 Tsukamurella (data not shown). The 15 Gordonia (comprising 8 G. sputi, 4 G. bronchialis, and 3 G. rubripertincta) isolates were distributed across nine provinces. Interestingly, the presence of Gordonia is consistent with a previous report from China [22]. Two Nocardia species, which often cause chronic lung disease, were isolated, as previously reported in China [23]. In addition, a case of Tsukamurella has previously been reported in Jiangxi province, Southern-central China [6]. In addition to NTM and MTBC infection, Gordonia and Nocardia species should also be tested for when using acid-fast staining to diagnosis pulmonary infection.

We evaluated the susceptibility of RGM and SGM from China to antimicrobials by measuring MIC values using the RAPIDMYCOI and SLOWMYCOI Sensititre ${ }^{\text {TM }}$ panels, according to CLSI protocol M24-A2. No such simple commercial kits for MIC measurement are available in China, despite the increase in patients with NTM infections, and information on drug susceptibility of NTM isolates is lacking. We mainly analyzed the susceptibility of MABC isolates, which were the most common clinical RGM isolates. Inducible macrolide resistance leads to differences in treatment outcome between patients with $M$. abscessus and M.massiliense infections. Consistent with previous reports [26-28], we found that M.abscessus had a higher inducible resistance $(65.67 \%$ vs $2.22 \%, p<0.01)$ and acquired resistance $(17.91 \%$ vs $8.89 \%, p=0.2841)$ rates for clarithromycin than M.massiliense. These results further emphasize the importance of M.abscessus and M.massiliense subspecies identification, to inform appropriate clinical treatment using different strategies. Amikacin was the most active antimicrobial agent against MABC species, showing a $94.74 \%$ overall susceptibility rate, similar to the overall susceptible rate observed in previous studies from China and Australia [29, 30]; however, higher resistance rates, from $28.2 \%$ to $76.0 \%$, have been observed in Japan and South Korea [15, 31]. After amikacin, cefoxitin was the second most effective antimicrobial agent against MABC, with a 16.67\% resistance rate, unlike results from South Korea [31], where the second most effective antimicrobial agent was linezolid, but consistent with findings from Japan [15]. The resistance rate to cefoxitin was higher in M.abscessus (19.40\%) than M.massiliense (11.11\%). Linezolid, with a resistance rate of $33.33 \%$, could be used as an alternative therapy choice against RGM isolates. Given the high resistance rates to the other drugs tested in our study, they may not be appropriate for treatment of MABC infections; however, studies of clinical therapeutic effects are required.

For SGM, we mainly analyzed the susceptibility of the MAC and M.kansasii, the two most frequent SGM species. Consistent with previous studies [32], macrolides and amikacin, as the first-line therapeutic agent for lung diseases caused by MAC infection, showed excellent in vitro activity against MAC isolates, with $90 \%$ susceptibility. Patients with MAC pulmonary diseases are frequently administered a combination of clarithromycin, ethambutol, and rifampicin; however, one study suggested that treatment with clarithromycin and ethambutol is not inferior to treatment with clarithromycin, ethambutol, and rifampicin for MAC lung disease [33]. Our data support the two treatment regimen, since the resistance rates to ethambutol and rifampicin in vitro were $58.33 \%$ and $91.67 \%$, respectively. Some researchers have reported differential drug susceptibility patterns of M.chimaera and other members of the MAC [34]. In our study, we only obtained one M.chimaera strain. We compared the drug susceptibility patterns of the two most frequent species of 
MAC, and found no significant difference between M. intracellulare and M.marseillense. As M.marseillense infections are rare in humans $[24,25,35]$, our drug susceptibility data add to knowledge of this species.

M. kansasii, which was the second most frequent SGM infection species identified in this study, showed a higher susceptibility rate to the majority of first- and second-line antibiotics recommended by CLSI (M24, 3rd Edition). These drug susceptibility patterns were markedly different from those reported by a previous study, which included DST of a total of $78 \mathrm{M}$. kansasii strains from 13 provinces of China [36]. Except for ethambutol (83.87\% vs $20.5 \%)$, the resistance rates in our study were lower than those reported by the previous study [36], as follows: clarithromycin (0 vs $20.5 \%$ ), amikacin (0 vs $5.1 \%$ ), rifampicin (6.45\% vs $56.4 \%$ ), rifabutin ( $3.23 \%$ vs $34.6 \%$ ), moxifloxacin ( 0 vs $16.7 \%$ ), and linezolid (3.23\%\% vs $32.1 \%$ ). Our results are similar to those of a study using $85 \mathrm{M}$. kansasii isolates from eight countries in Europe and Asia [37]. In this study, all $85 \mathrm{M}$. kansasii isolates were susceptible to rifampicin, amikacin, rifabutin, moxifloxacin, and linezolid. Although all $31 \mathrm{M}$. kansasii isolates included in our study were from 13 provinces in China, more isolates should be tested to evaluate drug susceptibility patterns of $\mathrm{M}$. kansasii, given the relatively small number of strains and regional disparities.

No NTM were identified from some provinces in the northwestern region, likely due to the small sample size. Also, there were a limited number of strains of each species. We have prepared to collect more samples from these regions to complete our analysis of NTM infection and drug resistance status in China, and plan to evaluate NTM infection using the isolates collected during nationwide surveillance of tuberculosis.

\section{Conclusions}

In this study, we analyzed the drug susceptibility patterns of all 317 NTM strains. More NTM pulmonary disease was observed in southern and coastal China $(p<0.01)$. SGM was widely distributed, and more RGM are present in southern and coastal China $(p<0.01)$. The antimicrobial resistance spectrum of different NTM species was significant different, accurate species identification would be facilitated to NTM pulmonary disease treatment. Understanding of NTM pulmonary infection will facilitate the development of TB treatment and control targets.

\section{List Of Abbreviations}

Nontuberculous mycobacteria (NTM)

National tuberculosis reference laboratory (NTRL)

Minimal inhibitory concentrations (MICs)

Slow growing mycobacteria (SGM)

Rapid growing mycobacteria (RGM)

NTM pulmonary disease (NTM-PD)

Mycobacterium tuberculosis (MTB)

Drug susceptibility testing (DST)

Lowenstein-Jensen (L-J)

Matrix-assisted laser desorption ionization-time of flight mass spectrometry (MALDI-TOF MS)

Clinical and Laboratory Standards Institute (CLSI)

Mycobacterium tuberculosis complex (MTBC)

Mycobacterium abscessus complex (MABC)

Mycobacterium avium-intracellulare complex (MAC) 


\section{Declarations}

\section{Ethics approval and consent to participate Contributions}

This study was approved by the ethics committee of the China Center for Disease Control and Prevention and conducted in accordance with the approved guidelines.

\section{Consent for publication}

Not applicable.

\section{Availability of data and materials}

The datasets used and/or analyzed during the current study are available from the corresponding author on reasonable request.

\section{Competing interests}

The authors declare that they have no competing interests.

\section{Funding}

This work was supported by the National Major Science and Technology Projects of China (2017ZX10304402-001-015), and the Chinese Center for Disease Control and Prevention Project (59911905).

\section{Authors' contributions}

Chunfa Liu and Yimeng Song were contributed to data collection and data analysis; Wencong He, Dongxin Liu, Ping He, Jingjing Bao and Wang Xinyang were participated in data collection; Yanming Li and Yanlin Zhao were worked for study design.

\section{Acknowledgements}

Not applicable.

\section{References}

1. Marras, T.K., et al., Isolation prevalence of pulmonary non-tuberculous mycobacteria in Ontario, 1997 2003. Thorax, 2007. 62(8): p. 661-666.

2. Wassilew, N., et al., Pulmonary Disease Caused by Non-Tuberculous Mycobacteria. Respiration, 2016. 91(5): p. $386-402$.

3. Johnson, C., et al., The Epidemiology and Geographic Distribution of Nontuberculous Mycobacteria Clinical Isolates from Sputum Samples in the Eastern Region of China. PLOS Neglected Tropical Diseases, 2015. 9(3): p. e0003623.

4. Zhou, L., et al., Trends in the Prevalence and Antibiotic Resistance of Non-tuberculous Mycobacteria in Mainland China, 2000-2019: Systematic Review and Meta-Analysis. Front Public Health, 2020. 8: p. 295.

5. Xu, J., et al., Prevalence and risk factors of pulmonary nontuberculous mycobacterial infections in the Zhejiang Province of China. Epidemiology and Infection, 2019. 147.

6. Zhou, D., et al., Identification and Characterization of Non-Tuberculous Mycobacteria Isolated from Tuberculosis Suspects in Southern-Central China. PLoS ONE, 2014. 9(12): p. e114353.

7. Yu, X., et al., The prevalence of non-tuberculous mycobacterial infections in mainland China: Systematic review and meta-analysis. Journal of Infection, 2016. 73(6): p. 558-567.

8. Alcaide, F., et al., How to: identify non-tuberculous Mycobacterium species using MALDI-TOF mass spectrometry. Clinical Microbiology and Infection, 2018. 24(6): p. 599-603.

9. Park, J.S., et al., The impact of protein extraction protocols on the performance of currently available MALDI-TOF mass spectrometry for identification of mycobacterial clinical isolates cultured in liquid media. Clinica Chimica Acta, 2016. 460: p. 190195.

10. Yanlin Zhao, P.D., Shaofa Xu, M.D., Lixia Wang, M.S., Daniel P. Chin, M.D.,, et al., National Survey of Drug-Resistant Tuberculosis in China. NEJM, 2012. 366: p. 2161-70. 
11. CLSI., Susceptibility Testing of Mycobacteria, Nocardia spp., and Other Aerobic Actinomycetes. 3rd ed. CLSI standard M24. Wayne, PA: Clinical and Laboratory Standards Institute; 2018.

12. SA, J., Two-sided confidence intervals for the single proportion: comparison of seven methods by Robert G. Newcombe, Statistics in Medicine 1998; 17:857-872. Statistics in medicine, 2005. 24(21): p. 3383-4.

13. Barbara A. Brown-Elliott, G.L.W., Antimycobacterial susceptibility testing of nontuberculous mycobacteria. J Clin Microbiol, 2019. 57(10): p. e00834-19.

14. Diel, R., et al., Burden of non-tuberculous mycobacterial pulmonary disease in Germany. European Respiratory Journal, 2017. 49(4): p. 1602109.

15. Aono, A., et al., Antimicrobial susceptibility testing of Mycobacteroides (Mycobacterium) abscessus complex, Mycolicibacterium (Mycobacterium) fortuitum, and Mycobacteroides (Mycobacterium) chelonae. Journal of Infection and Chemotherapy, 2019. 25(2): p. 117-123.

16. Thomson, R.M. and W.-W. Yew, When and how to treat pulmonary non-tuberculous mycobacterial diseases. Respirology, 2009. 14(1): p. 12-26.

17. Hui Jing, H.W., Yan Wang, Yunfeng Deng,Xinxin Li, Zhimin Liu, Edward A. Graviss, and Xin Ma, Prevalence of Nontuberculous Mycobacteria Infection, China, 2004-2009. Emerging Infectious Diseases, 2012. 18: p. 527-528.

18. Akos Somoskovi, J.M., Yvonne M. Hale, Linda M. Parsons, Max Salfinger, MD, Laboratory diagnosis of nontuberculous mycobacteria. Clin Chest Med, 2002. 23: p. 585-597.

19. Luo, L., et al., Evaluation of the VITEK MS knowledge base version 3.0 for the identification of clinically relevant Mycobacterium species. Emerging Microbes \& Infections, 2019. 7(1): p. 1-8.

20. Haworth, C.S. and R.A. Floto, Introducing the new BTS Guideline: Management of non-tuberculous mycobacterial pulmonary disease (NTM-PD). Thorax, 2017. 72(11): p. 969-970.

21. Jie Wu, Y.Z., Jing Li, Senlin Lin, Lili Wang, Yuan Jiang, Qichao Pan*, Xin Shen*, Increase in Nontuberculous Mycobacteria Isolated in Shanghai, China: Results from a Population-Based Study. PLoS ONE, 10-16, 2014. 9(10): p. e109736.

22. Pang, Y., et al., Diversity of nontuberculous mycobacteria in eastern and southern China: a cross-sectional study. European Respiratory Journal, 2017. 49(3): p. 1601429.

23. Liu, H., et al., Identification of Species of Nontuberculous Mycobacteria Clinical Isolates from 8 Provinces of China. BioMed Research International, 2016. 2016: p. 1-10.

24. SY, K., et al., First case of nontuberculous mycobacterial lung disease caused by Mycobacterium marseillense in a patient with systemic lupus erythematosus. Diagnostic microbiology and infectious disease, 2014. 79(3): p. 355-7.

25. A, G., et al., Pulmonary disease caused by Mycobacterium marseillense, Italy. Emerging infectious diseases, 2014. 20(10): p. 176970.

26. EH, C., et al., Drug susceptibility patterns of Mycobacterium abscessus and Mycobacterium massiliense isolated from respiratory specimens. Diagnostic microbiology and infectious disease, 2019. 93(2): p. 107-111.

27. D, M., et al., Mycobacterium abscessus In VitroVancomycin and Clarithromycin Show Synergy against. Antimicrobial agents and chemotherapy, 2017. 61(12).

28. SH, L., et al., Detection and assessment of clarithromycin inducible resistant strains among Korean Mycobacterium abscessus clinical strains: PCR methods. Journal of clinical laboratory analysis, 2014. 28(5): p. 409-14.

29. KY, C., et al., Antibiotic susceptibility of diverse Mycobacterium abscessus complex strains in New South Wales, Australia. Pathology, 2015. 47(7): p. 678-82.

30. Pang, H., et al., Drug Susceptibility Testing of 31 Antimicrobial Agents on Rapidly Growing Mycobacteria Isolates from China. Biomed Res Int, 2015. 2015: p. 419392.

31. Cho, E.H., et al., Drug susceptibility patterns of Mycobacterium abscessus and Mycobacterium massiliense isolated from respiratory specimens. Diagnostic Microbiology and Infectious Disease, 2019. 93(2): p. 107-111.

32. Zheng, H.W., et al., Comparing the Genotype and Drug Susceptibilities between Mycobacterium avium and Mycobacterium intracellulare in China. Biomed Environ Sci, 2017. 30(7): p. 517-525.

33. Miwa, S., et al., Efficacy of clarithromycin and ethambutol for Mycobacterium avium complex pulmonary disease. A preliminary study. Ann Am Thorac Soc, 2014. 11(1): p. 23-9. 
34. Maurer, F.P., et al., Differential drug susceptibility patterns of Mycobacterium chimaera and other members of the Mycobacterium avium-intracellulare complex. Clin Microbiol Infect, 2019. 25(3): p. 379 e1-379 e7.

35. B, X., et al., Mycobacterium marseillense Infection in Human Skin, China, 2018. Emerging infectious diseases, 2019. 25(10): p. 19911993.

36. Li, Y., et al., Mycobacterium kansasii Subtype I Is Associated With Clarithromycin Resistance in China. Front Microbiol, 2016. 7: p. 2097.

37. Bakula, Z., et al., Drug Susceptibility Profiling and Genetic Determinants of Drug Resistance in Mycobacterium kansasii. Antimicrob Agents Chemother, 2018. 62(4).

\section{Tables}

Table 1

Prevalence of NTM infection in different regions based on geographic areas of China

\begin{tabular}{|c|c|c|c|c|c|c|}
\hline \multirow[t]{2}{*}{ Regions } & \multirow[t]{2}{*}{ Isolates } & \multicolumn{2}{|l|}{ NTM } & \multicolumn{2}{|l|}{ NTM Rate $(95 \% \mathrm{Cl})$} & \multirow[t]{2}{*}{ p-value } \\
\hline & & RGM & SGM & RGM Rate $(95 \% \mathrm{Cl})$ & SGM Rate $(95 \% \mathrm{Cl})$ & \\
\hline \multirow[t]{2}{*}{ South } & 3709 & 287 & & $7.7(6.9-8.6)$ & & $<0.01$ \\
\hline & & 126 & 161 & $43.9(38.3-49.7)$ & $56.1(50.3-61.7)$ & $<0.01$ \\
\hline \multirow[t]{2}{*}{ North } & 1208 & 30 & & $2.5(1.7-3.5)$ & & \\
\hline & & 2 & 28 & $6.7(1.9-2.1)$ & 93.3 (78.7-98.2) & \\
\hline
\end{tabular}

Provinces locate in northern region: Hebei, Beijing, Tianjin, Liaoning, Shanxi, Shandong, Henan, Shaanxi, Inner Mongolia, Jilin, Heilongjiang, Tibet, Gansu, Qinghai, Ningxia, Xinjiang;

Provinces locate in northwestern region: Yunnan, Sichuan, Guizhou, Chongqing, Shanghai, Jiangsu, Zhejiang, Fujian, Anhui, Jiangxi, Hunan, Hubei, Guangxi, Guangdong, Hainan.

\begin{tabular}{|c|c|c|c|c|c|c|}
\hline \multirow[t]{2}{*}{ Coastal } & \multirow[t]{2}{*}{2207} & \multicolumn{2}{|l|}{236} & \multicolumn{2}{|l|}{$10.7(9.5-12.1)$} & \multirow{2}{*}{$\begin{array}{l}<0.01 \\
<0.01\end{array}$} \\
\hline & & 118 & 118 & $50.0(43.7-56.3)$ & $50.0(43.7-56.3)$ & \\
\hline \multirow[t]{2}{*}{ Inland } & 2710 & 81 & & $3.0(2.4-3.7)$ & & \\
\hline & & 10 & 71 & $12.4(6.9-21.3)$ & $87.7(78.7-93.2)$ & \\
\hline
\end{tabular}

Provinces locate in coastal region: Tianjin, Liaoning, Shanghai, Jiangsu, Zhejiang, Fujian, Shandong, Guangdong, Guangxi, Hainan, Hebei,

Provinces locate in inland region: Beijing, Shanxi, Inner Mongolia, Jilin, Heilongjiang, Anhui, Jiangxi, Henan, Hubei, Hunan, Chongqing, Sichuan, Guizhou, Yunnan, Tibet, Shaanxi, Gansu, Qinghai, Ningxia, Xinjiang 
Table 2

Susceptibility of RGM to antimicrobial agents determined by the broth dilution method

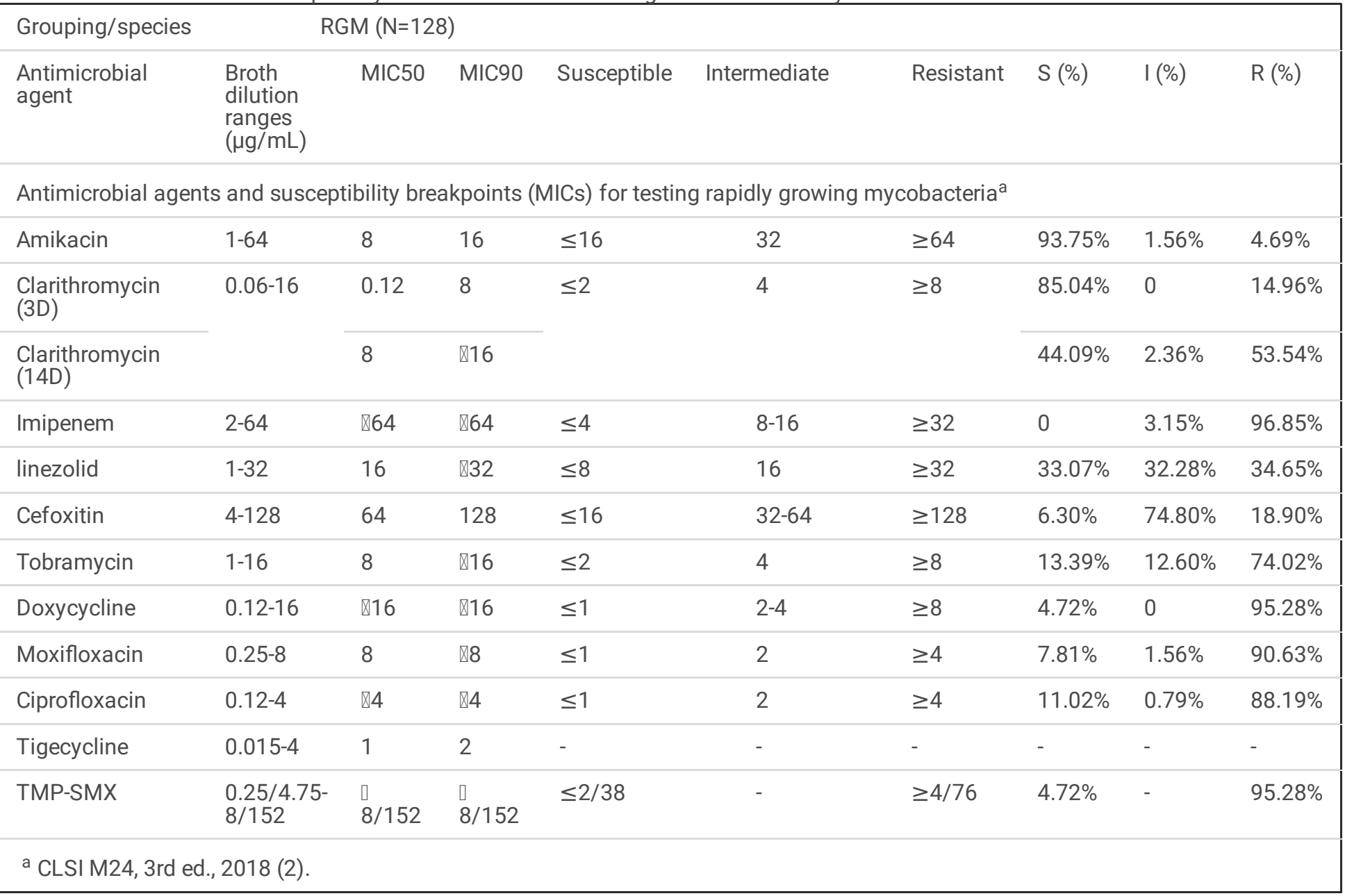


Table 3

Susceptibility of M. abscessus and M. massiliense to antimicrobial agents determined by the broth dilution method

\begin{tabular}{|c|c|c|c|c|c|c|c|c|c|}
\hline \multirow{2}{*}{$\begin{array}{l}\text { Grouping/species } \\
\text { Antimicrobial agent }\end{array}$} & \multicolumn{3}{|c|}{ M. abscessus complex $(\mathrm{N}=114)$} & \multicolumn{3}{|c|}{ M. abscessus $(n=67)$} & \multicolumn{3}{|c|}{ M. massiliense $(n=45)$} \\
\hline & S (\%) & I (\%) & $\mathrm{R}(\%)$ & S (\%) & I (\%) & $\mathrm{R}(\%)$ & S (\%) & I (\%) & $\mathrm{R}(\%)$ \\
\hline Amikacin & $94.74 \%$ & $1.75 \%$ & $3.51 \%$ & $92.54 \%$ & $1.49 \%$ & $5.97 \%$ & $100.00 \%$ & $0.00 \%$ & $0.00 \%$ \\
\hline Clarithromycin (3D) & $85.09 \%$ & $0.00 \%$ & $14.91 \%$ & $82.09 \%$ & $0.00 \%$ & $17.91 \%$ & $91.11 \%$ & $0.00 \%$ & $8.89 \%$ \\
\hline Clarithromycin (14D) & $42.98 \%$ & $2.63 \%$ & $54.39 \%$ & $13.43 \%$ & $2.99 \%$ & $83.58 \%^{a}$ & $86.67 \%$ & $2.22 \%$ & $11.11 \%^{a}$ \\
\hline Imipenem & $0.00 \%$ & $1.75 \%$ & $98.25 \%$ & $0.00 \%$ & $0.00 \%$ & $100.00 \%$ & $0.00 \%$ & $2.22 \%$ & $97.78 \%$ \\
\hline linezolid & $32.46 \%$ & $34.21 \%$ & $33.33 \%$ & $29.85 \%$ & $34.33 \%$ & $35.82 \%$ & $35.56 \%$ & $35.56 \%$ & $28.89 \%$ \\
\hline Cefoxitin & $4.39 \%$ & $78.95 \%$ & $16.67 \%$ & $4.48 \%$ & $76.12 \%$ & $19.40 \%$ & $4.44 \%$ & $84.44 \%$ & $11.11 \%$ \\
\hline Tobramycin & $11.40 \%$ & $12.28 \%$ & $76.32 \%$ & $8.96 \%$ & $10.45 \%$ & $80.60 \%$ & $13.33 \%$ & $15.56 \%$ & $71.11 \%$ \\
\hline Doxycycline & $1.75 \%$ & $0.00 \%$ & $98.25 \%$ & $1.49 \%$ & $0.00 \%$ & $98.51 \%$ & $2.22 \%$ & $0.00 \%$ & $97.78 \%$ \\
\hline Moxifloxacin & $2.63 \%$ & $1.75 \%$ & $95.61 \%$ & $1.49 \%$ & $1.49 \%$ & $97.01 \%$ & $2.22 \%$ & $0.00 \%$ & $97.78 \%$ \\
\hline Ciprofloxacin & $5.26 \%$ & $0.88 \%$ & $93.86 \%$ & $5.97 \%$ & $0.00 \%$ & $94.03 \%$ & $4.44 \%$ & $0.00 \%$ & $95.56 \%$ \\
\hline Tigecycline & - & - & - & - & - & - & - & - & - \\
\hline TMP-SMX & $2.63 \%$ & - & $97.37 \%$ & $2.99 \%$ & - & $97.01 \%$ & $2.22 \%$ & $0.00 \%$ & $97.78 \%$ \\
\hline
\end{tabular}

Table 4

Susceptibility of MAC to antimicrobial agents determined by the broth dilution method

Grouping/species M. avium-intracellulare complex $(\mathrm{N}=108)$ $\begin{array}{llllllll}\begin{array}{l}\text { Antimicrobial } \\ \text { agent }\end{array} & \begin{array}{l}\text { Broth dilution } \\ \text { range }(\mu \mathrm{g} / \mathrm{mL})\end{array} & \text { MIC50 } & \text { MIC90 } & \text { Susceptible } & \text { Intermediate } & \text { Resistant } & \text { S (\%) }\end{array}$

Antimicrobial agents and susceptibility breakpoints (MICs) for testing MAC ${ }^{a}$

\section{First line}

\begin{tabular}{|c|c|c|c|c|c|c|c|c|c|}
\hline Clarithromycin & $0.06-64$ & 2 & 4 & $\leq 8$ & 16 & $\geq 32$ & $95.37 \%$ & 0 & $4.63 \%$ \\
\hline Amikacin & $1-64$ & 16 & 64 & $\leq 16$ & 32 & $\geq 64$ & $71.30 \%$ & $18.52 \%$ & $10.19 \%$ \\
\hline \multicolumn{10}{|l|}{ Second line } \\
\hline Linezolid & $1-64$ & 32 & 64 & $\leq 8$ & 16 & $\geq 32$ & $14.81 \%$ & $18.52 \%$ & $66.67 \%$ \\
\hline Moxifloxacin & $0.12-8$ & 2 & 8 & $\leq 1$ & 2 & $\geq 4$ & $15.74 \%$ & $37.04 \%$ & $47.22 \%$ \\
\hline
\end{tabular}


Table 5

Susceptibility of M. kansasii to antimicrobial agents determined by the broth dilution method

\begin{tabular}{|c|c|c|c|c|c|c|c|c|c|}
\hline \multirow{2}{*}{$\begin{array}{l}\text { Grouping/species } \\
\text { Antimicrobial } \\
\text { agent }\end{array}$} & \multicolumn{9}{|c|}{ M. Kansasii $(\mathrm{N}=31)$} \\
\hline & $\begin{array}{l}\text { Broth dilution } \\
\text { ranges } \\
(\mu \mathrm{g} / \mathrm{mL})\end{array}$ & MIC50 & MIC90 & Susceptible & Intermediate & Resistant & $\mathrm{S}(\%)$ & I (\%) & $\mathrm{R}(\%)$ \\
\hline \multicolumn{10}{|c|}{ Antimicrobial agents and susceptibility breakpoints (MICs) for testing M. kansasii a } \\
\hline \multicolumn{10}{|l|}{ First line } \\
\hline Clarithromycin & $0.06-64$ & 0.25 & 0.5 & $\leq 8$ & 16 & $\geq 32$ & $100 \%$ & 0 & 0 \\
\hline Rifampicin & $0.12-8$ & 0.5 & 0.5 & $\leq 1$ & - & $\geq 2$ & $93.55 \%$ & - & $6.45 \%$ \\
\hline \multicolumn{10}{|l|}{ Second line } \\
\hline Amikacin & $1-64$ & 2 & 8 & $\leq 16$ & 32 & $\geq 64$ & $96.77 \%$ & $3.23 \%$ & 0 \\
\hline Ciprofloxacin & $0.12-16$ & 2 & 4 & $\leq 1$ & 2 & $\geq 4$ & $32.26 \%$ & $41.93 \%$ & $25.81 \%$ \\
\hline Doxycycline & $0.12-16$ & 8 & 16 & $\leq 1$ & $2-4$ & $\geq 8$ & $9.68 \%$ & $12.90 \%$ & $77.42 \%$ \\
\hline Linezolid & $1-64$ & 2 & 4 & $\leq 8$ & 16 & $\geq 32$ & $96.77 \%$ & 0 & $3.23 \%$ \\
\hline Moxifloxacin & $0.12-8$ & $\leq 0.12$ & 0.25 & $\leq 1$ & 2 & $\geq 4$ & $96.77 \%$ & $3.23 \%$ & 0 \\
\hline Rifabutin & $0.25-8$ & $\leq 0.25$ & 0.5 & $\leq 2$ & - & $\geq 4$ & $96.77 \%$ & 0 & $3.23 \%$ \\
\hline TMP-SMX & $\begin{array}{l}0.12 / 2.38- \\
8 / 152\end{array}$ & $4 / 76$ & $\begin{array}{l}0 \\
8 / 152\end{array}$ & $\leq 2 / 38$ & - & $\geq 4 / 76$ & $48.39 \%$ & - & $51.61 \%$ \\
\hline
\end{tabular}

Table 6

Susceptibility of SGM other than MAC and M. kansasii to antimicrobial agents determined by the broth dilution method Grouping/species SGM other than MAC and M. kansasii $(\mathrm{N}=50)$

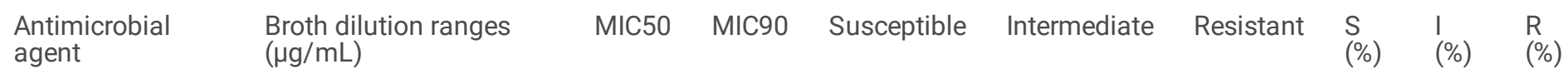

Antimicrobial agents and susceptibility breakpoints (MICs) for testing SGM other than MAC and M. kansasii a

\begin{tabular}{|c|c|c|c|c|c|c|c|c|c|}
\hline Amikacin & $1-64$ & 4 & 32 & $\leq 16$ & 32 & $\geq 64$ & $86 \%$ & $6 \%$ & $8 \%$ \\
\hline Ciprofloxacin & $0.12-16$ & 4 & $>16$ & $\leq 1$ & 2 & $\geq 4$ & $12 \%$ & $24 \%$ & $64 \%$ \\
\hline Clarithromycin & $0.06-64$ & 0.5 & 4 & $\leq 8$ & 16 & $\geq 32$ & $94 \%$ & 0 & $6 \%$ \\
\hline Doxycycline & $0.12-16$ & $>16$ & $>16$ & $\leq 1$ & $2-4$ & $\geq 8$ & $8 \%$ & $10 \%$ & $82 \%$ \\
\hline Linezolid & $1-64$ & 8 & 64 & $\leq 8$ & 16 & $\geq 32$ & $58 \%$ & $4 \%$ & $38 \%$ \\
\hline Moxifloxacin & $0.12-8$ & 2 & $>8$ & $\leq 1$ & 2 & $\geq 4$ & $50 \%$ & $24 \%$ & $26 \%$ \\
\hline Rifampicin & $0.12-8$ & 2 & $>8$ & $\leq 2$ & - & $\geq 4$ & $56 \%$ & - & $44 \%$ \\
\hline Rifabutin & $0.25-8$ & 0.5 & 1 & $\leq 1$ & - & $\geq 2$ & $96 \%$ & - & $4 \%$ \\
\hline TMP-SMX & $0.12 / 2.38-8 / 152$ & 2 & $>8$ & $\leq 2 / 38$ & - & $\geq 4 / 76$ & $46 \%$ & - & $54 \%$ \\
\hline
\end{tabular}


Percentage of drug resistance among different species of SGM.

\begin{tabular}{|c|c|c|c|c|c|c|}
\hline \multirow{3}{*}{$\begin{array}{l}\text { Grouping/species } \\
\text { Antimicrobial } \\
\text { agent }\end{array}$} & \multicolumn{2}{|l|}{ MAC } & \multicolumn{2}{|l|}{ M. kansasii } & \multicolumn{2}{|c|}{ Other SGM } \\
\hline & \multirow[t]{2}{*}{$\mathrm{R}(\%, \mathrm{n} / \mathrm{N})$} & $\begin{array}{l}\text { MAC vs M. } \\
\text { kansasii }\end{array}$ & \multirow[t]{2}{*}{$\mathrm{R}(\%, \mathrm{n} / \mathrm{N})$} & $\begin{array}{l}\text { M. kansasii vs Other } \\
\text { SGM }\end{array}$ & \multirow[t]{2}{*}{$\begin{array}{l}\mathrm{R}(\%, \\
\mathrm{n} / \mathrm{N})\end{array}$} & $\begin{array}{l}\text { MAC vs Other } \\
\text { SGM }\end{array}$ \\
\hline & & p Value & & p Value & & p Value \\
\hline Amikacin & $\begin{array}{l}10.19 \\
(11 / 108)\end{array}$ & 0.12 & $0(0 / 31)$ & 0.16 & $8(4 / 50)$ & 0.88 \\
\hline Clarithromycin & $4.63(5 / 108)$ & 0.35 & $0(0 / 31)$ & 0.28 & $6(3 / 50)$ & 1.00 \\
\hline Linezolid & $\begin{array}{l}66.67 \\
(72 / 108)\end{array}$ & $<0.01^{\mathrm{a}}$ & $\begin{array}{l}3.23 \\
(1 / 31)\end{array}$ & $<0.01^{\mathrm{a}}$ & $\begin{array}{l}38 \\
(19 / 50)\end{array}$ & $<0.01^{\mathrm{a}}$ \\
\hline Moxifloxacin & $\begin{array}{l}47.22 \\
(51 / 108)\end{array}$ & $<0.01^{a}$ & $0(0 / 31)$ & $<0.01^{\mathrm{a}}$ & $\begin{array}{l}26 \\
(13 / 50)\end{array}$ & 0.01 \\
\hline
\end{tabular}

\section{Figures}

Acid-fast bacilli (AFB)-positive tuberculous suspects from 72 stations of nationwide surveillance of tuberculosis in 2013

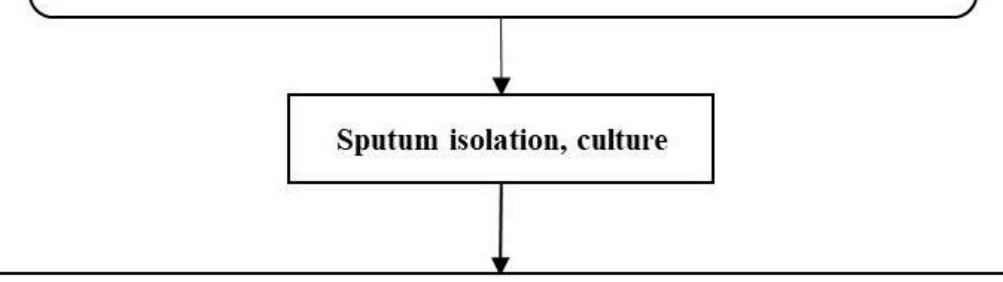

Culture positive isolations were transported to the national reference laboratory, $\mathrm{N}=\mathbf{5 4 8 7}$

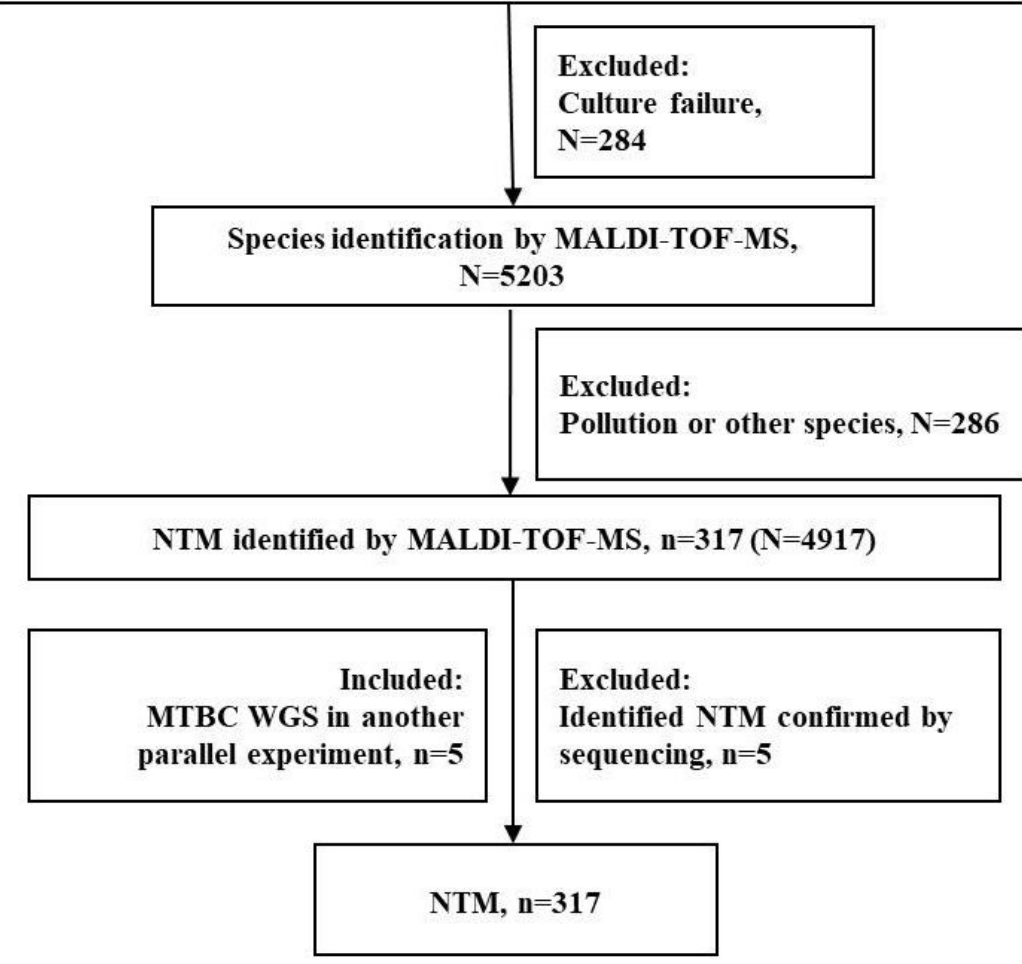


Figure 1

Flow chart

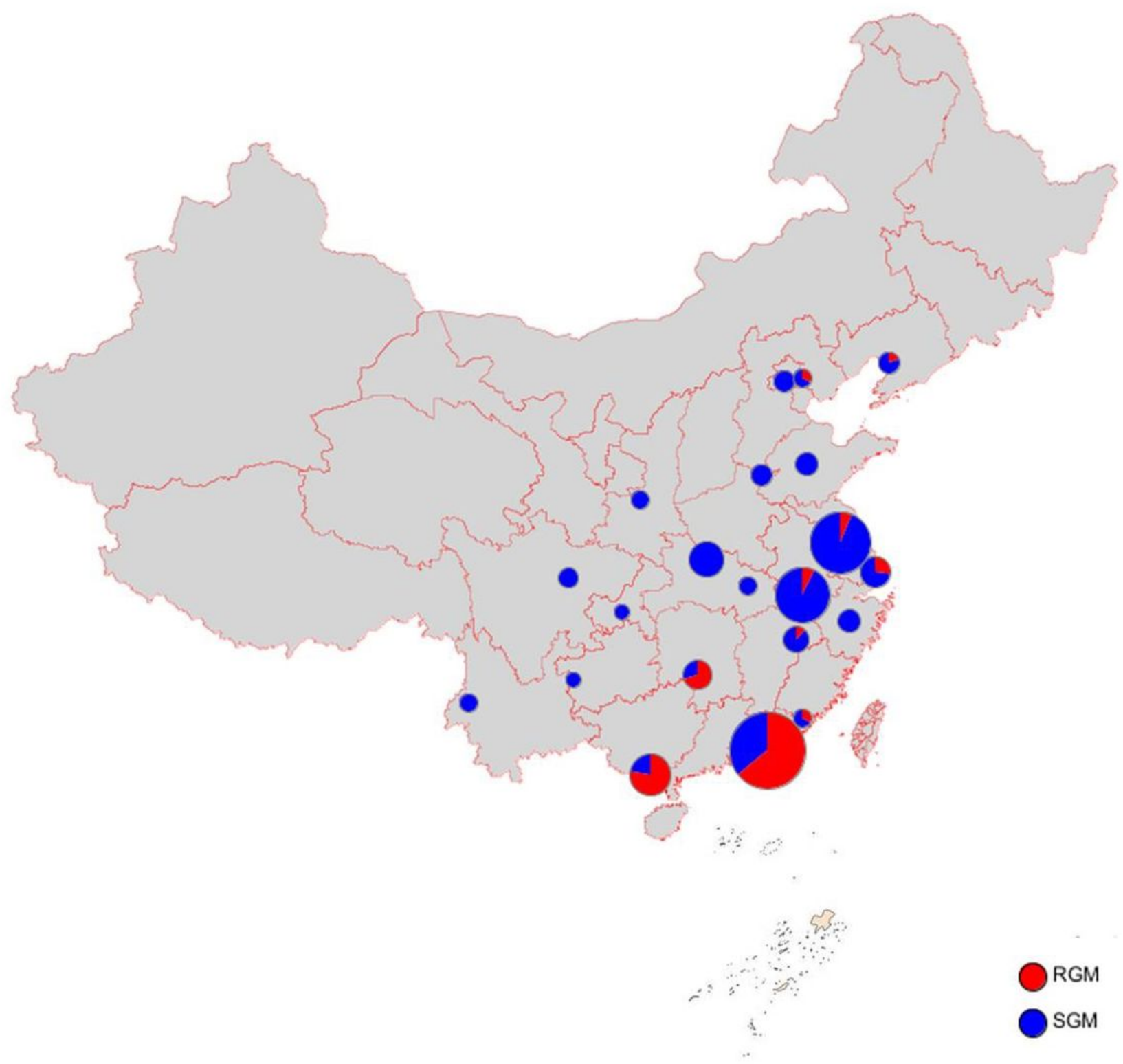

Figure 2

Prevalence rates in individual provinces. The composition ratio of RGM to SGM in northern and southern China differed markedly ( $p<$ 0.01). Note: The designations employed and the presentation of the material on this map do not imply the expression of any opinion whatsoever on the part of Research Square concerning the legal status of any country, territory, city or area or of its authorities, or concerning the delimitation of its frontiers or boundaries. This map has been provided by the authors. 
= Species

category

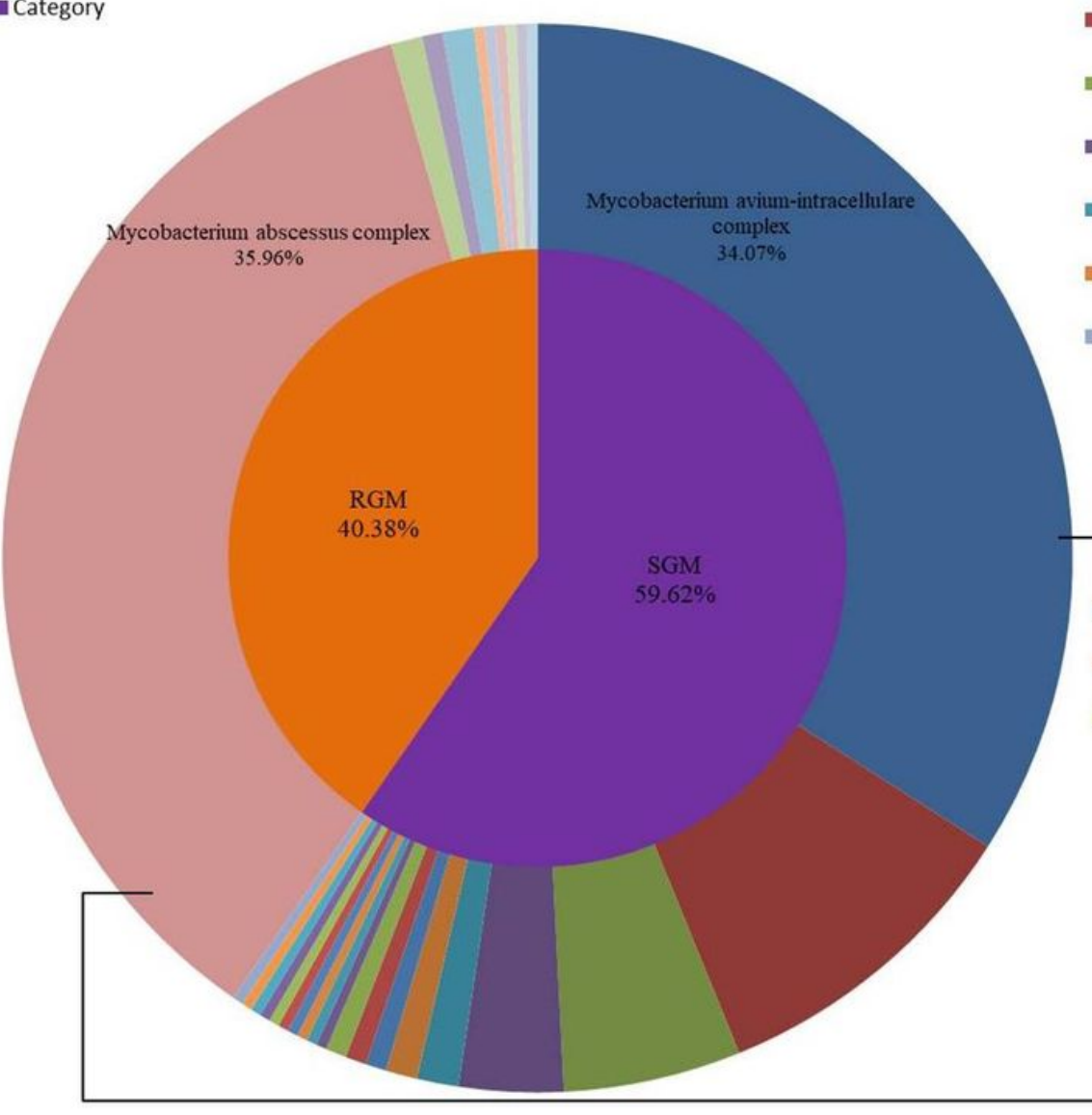

- Mycobacterium chimaera

Mycobacterium avium subsp.hominissuis

Mycobacterium avium subsp.vulneris

Mycobacterium avium

Mycobacterium avium subsp. Marseillense

Mycobacterium intracellulare

Mycobacterium intracellulare

subsp.yongonense

Mycobacterium bolletii

nycobacterium massiliease

\section{Figure 3}

Spectrum of NTM species

\section{Supplementary Files}

This is a list of supplementary files associated with this preprint. Click to download.

- GraphicalAbstractlmage.jpg 